\title{
Cigarette smoking and acute myocardial infarction: a case-control study from Argentina
}

H Schargrodsky, J Rosloznik, M Ciruzzi, P Pramparo, A Hirschon Prado, J Cesar, $S$ Soifer, E Negri, G Tognoni, C La Vecchia

\begin{abstract}
Objective - To assess the relationship between cigarette smoking and the risk of acute myocardial infarction in Argentina.
\end{abstract}

Design-Hospital-based case-control study conducted between 1984 and 1989 in Buenos Aires. Main outcome measures were multiple logistic regression analysis of relative risk (RR) estimates, and the corresponding $95 \%$ confidence intervals (CI), according to smoking status. Allowance was made for sex, age, education, body mass index, diabetes, hypertension, and family history of coronary heart disease.

Setting - A network including 11 coronary care units from major hospitals in Buenos Aires.

Subjects - Cases were 1000 patients with acute myocardial infarction. Controls were 1000 patients admitted to the same network of hospitals with a wide spectrum of acute disorders unrelated to smoking or to known or suspected risk factors for myocardial infarction.

Results - After allowance for major risk factors for myocardial infarction, compared to lifelong non-smokers, the RRs were $1.6(95 \% \mathrm{CI}=1.1$ to 2.4$)$ for current smokers of less than 15 cigarettes per day, $2.2(95 \% \mathrm{CI}=1.6$ to 3.0$)$ for smokers of $15-24$, and $5.9(95 \% \mathrm{CI}=4.2$ to 8.3$)$ for smokers of 25 or more cigarettes per day. The RR was $1.5(95 \% \mathrm{CI}=1.1$ to 2.0$)$ for ex-smokers who had been abstinent for at least one year. The RR at younger age (30-44 years) was 10.3 (95\% CI $=4.3$ to 24.9) for heavy smokers ( $\geqslant 25$ cigarettes per day), and declined with advancing age.

Conclusions-Cigarette smoking is an independent and important risk factor for acute myocardial infarction, and about $40 \%$ of cases in this Argentinian population (and over $55 \%$ of cases under age 55) are attributable to it.

(Tobacco Control 1993; 2: 127-131)

\section{Introduction}

The association between cigarette smoking and acute myocardial infarction (AMI) has been extensively investigated in several cohort and case-control studies conducted in North
America and Europe. ${ }^{1}$ Although a positive relationship has been consistently observed the strength of the association has been variable across studies, sex, and age groups. ${ }^{1-12}$ Thus, the relative and absolute role of smoking on myocardial infarction might be quantitatively different in other populations and areas of the world from which only scanty data are available, and where risk factors might weigh differently in the causation of cardiovascular events, with correspondingly variable public health implications.

Coronary heart disease is moderately frequent in Argentina. The overall age-adjusted (on the world standard population) mortality rate, for all ischaemic heart disease (International Classification of Diseases (ICD), 9th Revision, codes 410-414), in 1985-89 was $90 / 100000$ males and 29/100000 females.

To investigate and quantify the role of tobacco and other major risk factors in AMI, we conducted a large case-control study in Buenos Aires, Argentina. The results reported here are a first contribution to the elucidation of risk factors for coronary disease, through a case-control surveillance strategy, which has been established on a pilot basis in Buenos Aires, Argentina. In a broader perspective such a study scheme could be extended to other settings of Latin America, where cardiovascular morbidity and mortality have a major public health importance.

\section{Subjects and methods}

The data were derived from a hospital-based case-control study conducted between 1984 and 1989 in a network including major coronary care units in the metropolitan area of Buenos Aires. Trained interviewers identified and questioned cases of acute myocardial infarction and controls admitted to hospital for a wide spectrum of acute conditions, unrelated to smoking and to known or potential risk factors for coronary heart disease.

A structured questionnaire was used to obtain information about socio-demographic habits, including occupation, personal characteristics and habits, physical activity, selfreported weight and height, history of diabetes and hypertension, and personal and family history of coronary heart disease. Questions on smoking included smoking status (never, current, ex-smokers), for current and ex-smokers the number of cigarettes smoked per day, the 
duration of smoking in years, and, for exsmokers, time since stopping. Subjects who had smoked regularly (at least one cigarette per day) for at least one year and had smoked during the previous year were considered current smokers. Ex-smokers were subjects who had not smoked in the last year but reported that they had smoked in the past. On average, less than $2 \%$ of cases and controls refused to be interviewed.

Cases were patients aged 30 to 65 years who had been admitted for a first episode of AMI (ICD, 9th Revision, code 410) to coronary care units of 11 hospitals of metropolitan Buenos Aires (capital and suburbs). They were eligible if they met the standard World Health Organisation criteria for definition of acute myocardial infarction, ${ }^{13}$ including pathological $Q$ waves with evolution, or any of the following: typical history of chest pain for at least 30 minutes plus other electrocardiographic changes with evolution, and elevated cardiac enzymes. A total of 1000 cases (873 males, 127 females) were interviewed. Exclusion was made of cases with previous episodes of ischaemic heart conditions, including established and suspected infarction and angina pectoris. The median age of the cases was 55 years.

Controls were subjects aged 30 to 65 who had been admitted to the same network of hospitals where cases had been identified, for a wide spectrum of acute conditions unrelated to known or potential risk factors for myocardial infarction and to cigarette smoking. The main diagnostic categories were traumatic conditions $(42 \%)$, surgical diseases $(35 \%)$, and other miscellaneous illnesses, such as skin, nose, ear, throat, or dental disorders $(23 \%)$. Although no single matching was performed, cases and controls were comparable with reference to sex, age, and hospital. For each hospital and calendar period (i.e., month), the same number of controls as cases interviewed was randomly identified and approached for interview within comparable strata of sex and age, among all potential eligible controls admitted to the same hospital. Patients with a history of AMI or other ischaemic heart diseases, including angina pectoris or cardiac surgery, were excluded. Also excluded were subjects admitted for neoplastic, cardio- and cerebrovascular disorders, or any chronic condition. This, however, applied only to the admission diagnosis. The median age of the controls was 54 years.

\section{DATA ANALYSIS}

Relative risks (RR) of myocardial infarction and the corresponding $95 \%$ confidence intervals (CI) according to various measures of cigarette smoking compared with never smokers were first computed using multiple logistic regression equations, including terms for age and sex only. ${ }^{14}$ Further, models were fitted including terms for sex, decade of age, education, Quetelet's index (Body Mass Index (BMI)), history of diabetes and hypertension, and family history of coronary heart disease. The significance of the linear trends in risk was assessed by comparing the difference in the deviance of the models with and without the variable of interest to the chi-square distribution with one degree of freedom.

\section{Results}

Table 1 gives the distribution of myocardial infarction cases and controls according to age, sex, and selected risk factors. The prevalence of diabetes, hypertension, overweight, and family history of coronary heart disease was higher in the case group. The multivariate relative risks were $2.3(95 \% \mathrm{CI}=1.8$ to 2.8$)$ for hypertension, $1.2(95 \% \mathrm{CI}=1.0$ to 1.5$)$ for overweight (BMI, $\mathrm{kg} \cdot \mathrm{m}^{-2} 25$ to $29 \mathrm{vs}<25$ ), $1.7(95 \% \mathrm{CI}=1.3$ to 2.4$)$ for severe obesity

Table 1 Distribution of 1000 cases of acute myocardial infarction and 1000 controls according to sex, age, hypertension, body mass index, diabetes, and family history of coronary heart disease. Buenos Aires, Argentina,
1984-89

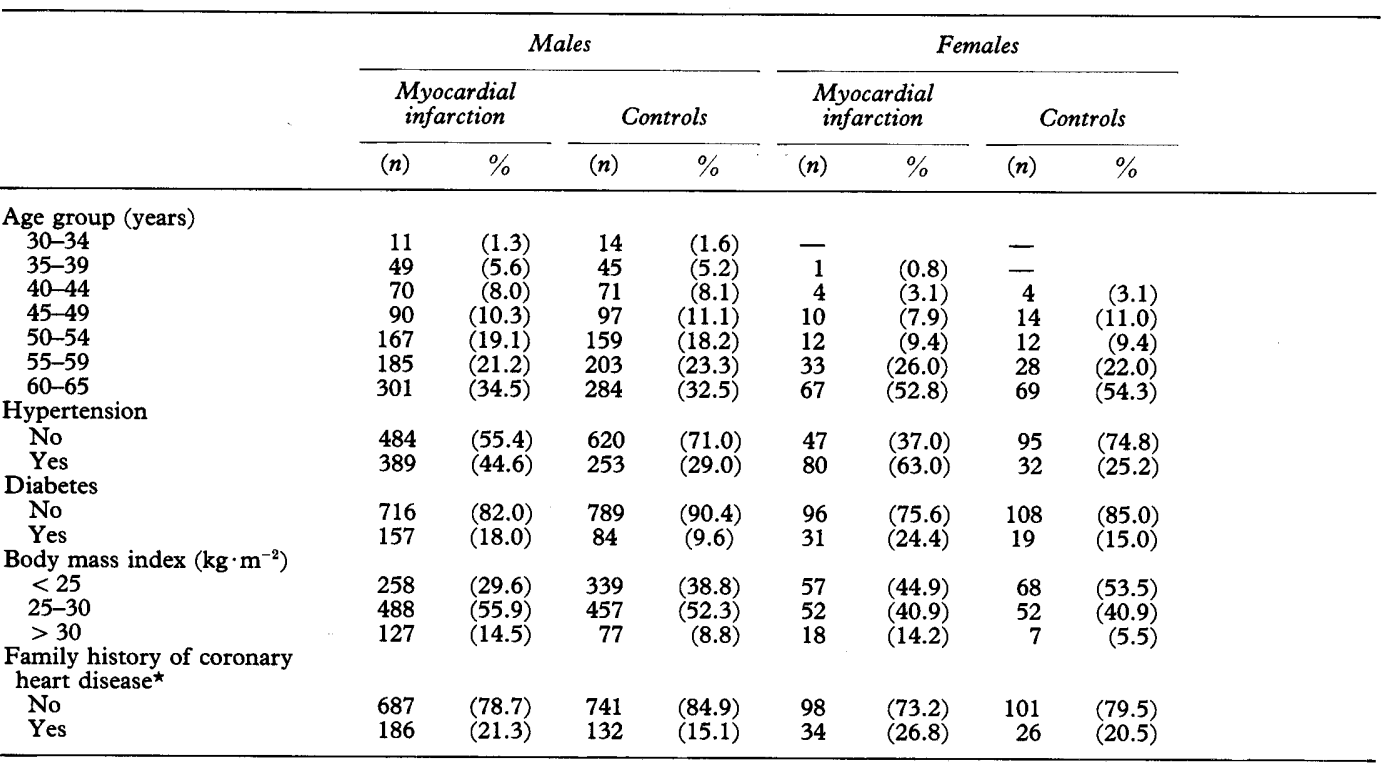

$\star$ Any coronary heart disease in first-degree relatives. 
Table 2 Distribution of 1000 cases of acute myocardial infarction and 1000 controls according to smoking status. Buenos Aires, Argentina 1984-89

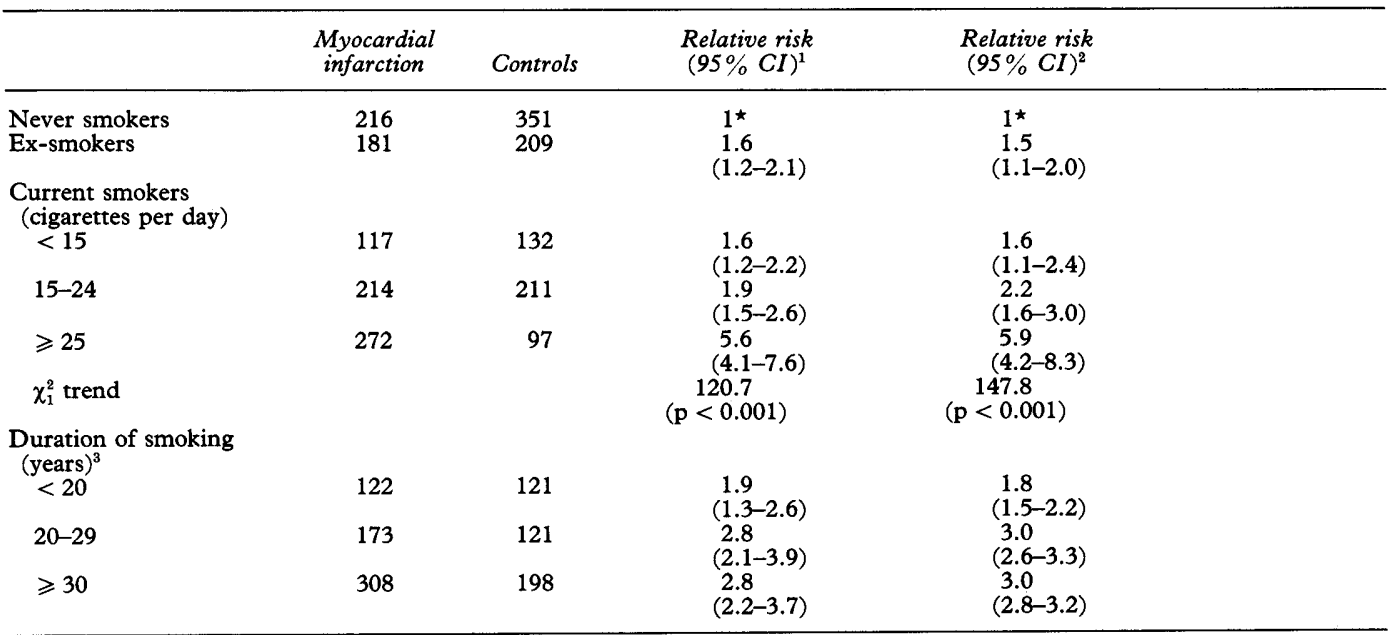

1 Estimates from multiple logistic regression equations including terms for age and sex.

2 Estimates from multiple logistic regression equations including terms for age, sex, diabetes, hypertension, body mass index, and family history of coronary heart disease.

Current smokers only.

* Reference category.

Table 3 Relative risk of acute myocardial infarction according to smoking status in separate strata of selected covariates. Buenos Aires, Argentina 1984-89

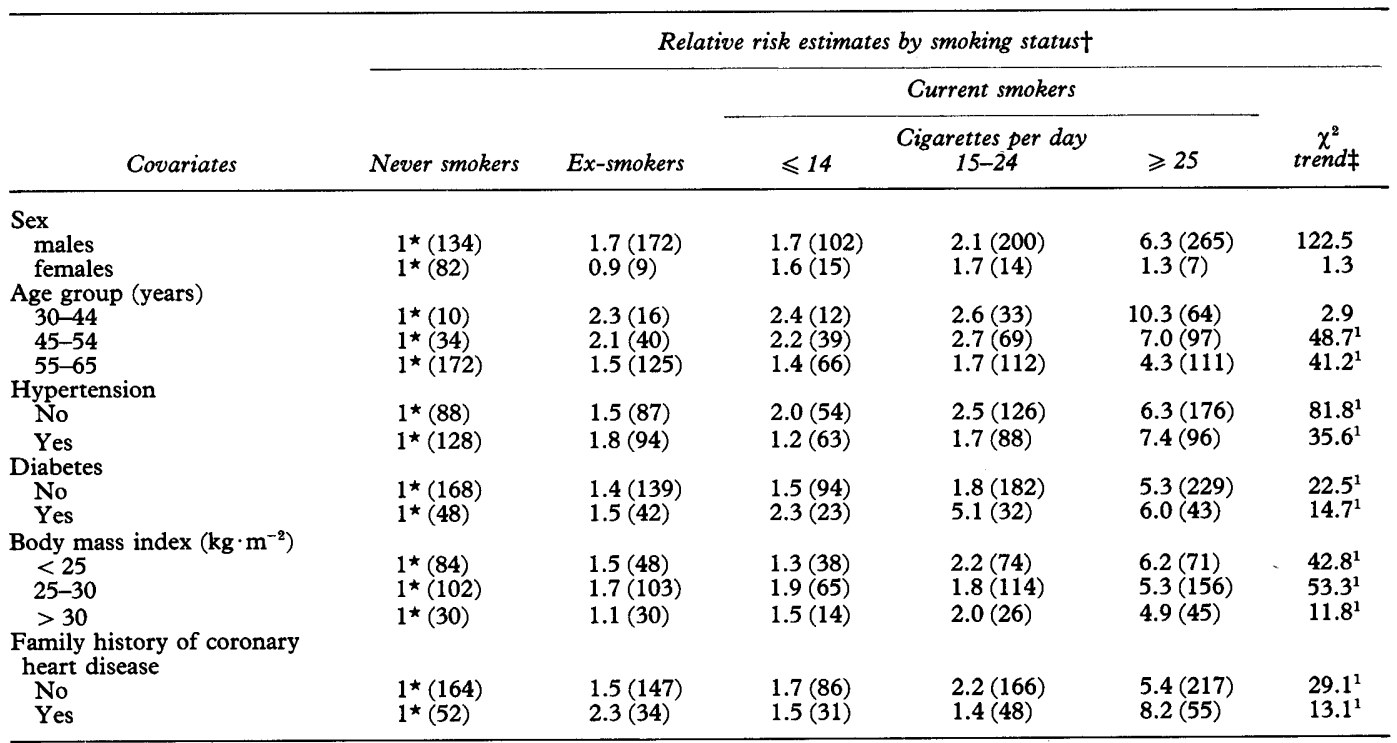

* Reference category

† Estimates from multiple logistic regression including terms for age and sex. Number of cases are given in parentheses. Ex-smokers excluded.

$\mathrm{p}<0.01$.

(BMI, $\mathrm{kg} \cdot \mathrm{m}^{-2} \geqslant 30$ vs $\left.<25\right), 2.0(95 \% \mathrm{CI}=$ 1.4-2.6) for diabetes, and $1.5(95 \%$ CI 1.2 to 1.9) for family history of coronary heart disease in first-degree relatives.

Smoking-related variables are considered in table 2. Twenty-one percent of cases versus $35 \%$ of the controls were lifelong nonsmokers. In comparison with them, the RR was moderately above unity in ex-smokers $(\mathrm{RR}=1.6,95 \% \mathrm{CI}=1.2$ to 2.1$)$. Among current smokers the RR was $1.6(95 \% \mathrm{CI}=1.2$ to 2.2 ) for less than 15 cigarettes per day, 1.9 ( $95 \% \mathrm{CI}=1.5$ to 2.6 ) for 15 to 24 cigarettes per day, and $5.6(95 \% \mathrm{CI}=4.1$ to 7.6$)$ for 25 or more cigarettes per day. The trend in risk for number of cigarettes per day was highly significant $\left(\chi_{1}^{2}=120.7, \mathrm{p}=0.001\right)$.

With reference to duration of smoking, the relative risk was 1.9 for less than 20 years, but there was no further trend of increasing risk for duration longer than 20 years $(R R=2.8$ for both 20 to 29 and for 30 years or more of smoking).

The risk estimates for current smokers were, if anything, higher after simultaneous allowance for diabetes, hypertension, BMI, and family history of coronary heart disease, besides age and sex: the $\mathrm{RR}$ was $1.6(95 \% \mathrm{CI}=$ 1.1 to 3.4$)$ among moderate, $2.2(95 \% \mathrm{CI}=1.6$ to 3.0$)$ among intermediate, and $5.9(95 \% \mathrm{CI}=$ 4.2 to 8.3 ) among heavy smokers.

The relationship between cigarette smoking and myocardial infarction is further examined in table 3 in separate strata of sex, age, and other major covariates. The risk estimates for current smokers were above unity in all the 
strata considered, but the association was apparently stronger in males than in females (although absolute numbers of female cases were low) and at younger than at older ages. The point estimate reached $10.3(95 \% \mathrm{CI}=$ 4.3 to 24.9 ) for heavy smokers aged 30 to 44 years. No apparent interaction was observed with any of the other risk factors considered.

\section{Discussion}

The present study confirms that cigarette smoking is a major determinant of ischaemic heart disease, and provides quantitative estimates of risk in an urban South American population. After allowance for major identified potential confounding factors, the risk was approximately two-fold higher in moderate smokers, and over five-fold higher in heavy smokers. The relative risk was apparently higher at younger age, reaching a factor of 10 for heavy smokers under age 45 . Still, the relative risk was over 4 for heavy smokers in late middle age (55 to 64).

This is a typical hospital-based case-control study and, as such, has all the related strengths and weaknesses. It is conceivable, for instance, that cigarette smoking may be associated with some of the diseases leading to hospital admission for the controls, ${ }^{15}$ and this would have caused an underestimation of the true risk. However, specific attention was paid to exclude all diagnoses known or thought to be related to cigarette smoking from the control group. Further, the multivariate relative risk of 5.9 observed in this study among heavy smokers is among the highest reported from the better conducted studies in the past, ${ }^{4,16-19}$ and it would be a posteriori surprising if a major underestimate was present in these data.

None of the smoking-related relative risks was appreciably modified by allowance for several potential distorting factors, including major determinants of ischaemic heart disease, using multivariate analysis. Although only dichotomous information was available on some of these factors, the multivariate relative risks for current smokers were higher than the age-adjusted ones, thus suggesting that even more accurate adjustment would be unlikely to account for the association observed. Further, no appreciable interaction emerged between smoking and any of the other major recognised risk factors for myocardial infarction. Among the strengths of the study, moreover, are the practically complete participation, the comparable catchment area of cases and controls, and the similarity of the results across major diagnostic categories of the controls (i.e., the proportion of current smokers ranged between 41 and $48 \%$ in various diagnostic categories).

The risk estimate was also somewhat elevated among ex-smokers. This is possibly attributable to the definition of ex-smokers in the study, simply as individuals who had been abstinent for at least one year. Although it is known that the risk of myocardial infarction for ex-smokers declines towards that of never smokers in a short period of time, it is possible that some residual excess risk is present for a few years after stopping. ${ }^{16-18}$ Alternatively, some misclassification is possible between exand current smokers, if some patients who defined themselves as ex-smokers were instead current smokers, or had been abstinent for only a very short time.

Possible explanations for the elevated risk in this study include chance (although the lower $95 \%$ confidence limit for heavy smokers in this study was still over 4), the age distribution with an age limit of 65 years (since the RR for smoking and myocardial infarction tends to be higher at younger ages), a precise definition of AMI, or different baseline characteristics of the population. Another plausible interpretation, however, is that the relative risks for current smokers tend to be systematically underestimated in some cohort studies, since some of the subjects classified as current smokers at interview may stop smoking during the follow-up period, and hence experience a substantial reduction of their risk of coronary heart disease. ${ }^{1,16}$ Case-control studies are less subject to this exposure classification bias, and should therefore offer a more accurate measure of risk for current smokers. The risk was similarly elevated in other case-control studies conducted in North America ${ }^{16-18}$ and Italy. ${ }^{20}$

In conclusion, therefore, this study confirms that smoking is a strong risk factor for AMI, particularly at younger ages. In this population from Buenos Aires, smoking accounted for about $40 \%$ of all cases of AMI and $56 \%$ of cases under age $55 .{ }^{21}$ These figures underline the importance of preventive intervention against smoking to control coronary heart disease in various areas of the world. Such intervention is particularly important and urgent in consideration of the recent trends in smoking prevalence in developing areas, quite different from the declines observed in industrialised countries. Even more important is to insist on the role of prevention, which will ultimately decrease the burden for already strained health systems.

This work was conducted with the contribution of Roemmers Laboratory and Behringwerke AG. We acknowledge the contribution of Ms Ivana Garimoldi, Perla Rafinet and Paola contribution of Ms Ivana Garimoldi, Perla Rafinet and Paola Bonifacino for their assistance in preparing this manuscript.
The authors are also indebted to Dr J Rimoldi, Dr R Raymondo The authors are also indebted to Dr J Rimoldi, Dr R Raymo

Participating Clinical Centers: Durand Hospital (A Cecchi), Ferroviario Hospital, Fiorito Hospital, Frances Hospital, (J Cesar), Israelita Hospital (S Soifer), Italiano Hospital (H Shargrodsky, N Vulcano, A. Caccavo), Mendez Hospital (C Paterno, R Ruffa, D Coria), Militar Hospital (M Ardariz), Paterno, R Ruffa, D Coria), Militar Hospital (M Ardariz),
Pirovano Hospital (M Ciruzzi), Posadas Hospital (J Rozlosnik, P Pramparo), Rivadavia Hospital (A Hirschon Prado).

1 US Office on Smoking and Health. The health consequences of smoking. Cardiovascular disease. Report of the Surgeon General. Rockville, MD, 1983.

2 Dorn HF. Tobacco consumption and mortality from cancer and other diseases. Public Health Rep $1959 ; 74: 581-93$.

3 Doll R, Gray R, Hafner B, Peto R. Mortality in relation. . doctors. $B M F$ 1980; 280: 967-71.

4 Doll R, Peto R. Mortality in relation to smoking: 20 Years' observations on male British doctors. BMF 1976; 2 : observation 5 Best EWRA. Canadian Study of Smoking and Health. Epidemiology Division, Health Services Branch, Biostatistics Division, Research and Statistics Directorate. 1966; 137pp.

6 Cederlof R, Friberg L, Hrubec Z, Lorich U. The relationship of Smoking and Some Social Covariables to Mortality and Cancer Morbidity. A ten-year follow-up in 
a probability sample of 55000 Swedish subjects, age 18-69, Parts 1 and 2. Stockholm, Sweden, Department of Environmental Hygiene, Karolinska Institute, 1975; $91 \mathrm{pp}$.

7 Hammond EC. Smoking in relation to the death rates of one million men and women. NCI Monogr 1966; 19: 127-204.

8 Hammond EC, Horn D. Smoking and death rates. Report on forty-four months of follow-up of 187783 men: II. on forty-four months of follow-up of 187783 men:

9 Hirayama T. Kitsuen to kyoketsusei shinzobyo to no kankei ni kansuru ekigakuteki kenkyu. [An epidemiological study
on smoking and ischemic heart disease.] Gen Clin $\mathcal{7} 1978$; on smoking

10 Rogot E, Murray JL. Smoking and causes of death among US veterans: 16 years' of observation. Public Health Rep $1980 ; 95: 213-22$.

11 Weir JM, Dunn JE Jr. Smoking and mortality: A prospective study. Cancer $1970 ; 25$ : 105-112.

12 Strobel M, Gsell O. Mortalitat in Bezeihung zum Tabakrauchen. 9 Jahre Beobachtungen bei Arzten in der Schweiz. [Mortality in relation to tobacco smoking. Nine years of observation in Swiss physicians]. Helv Med Acta $1965 ; 32: 547-92$

13 Rose GA, Blackburn H. Cardiovascular survey methods. 2nd edn World Health Organisation, Geneva, 1982.

$\mathbf{A}$

$-$

$\checkmark$

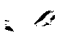

a

, is

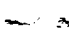

c

5

$\cdots$

-

$\pi$

$\therefore$

$=x$

2

$-$

$y$

$\therefore=$

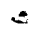

.

$\cdots$
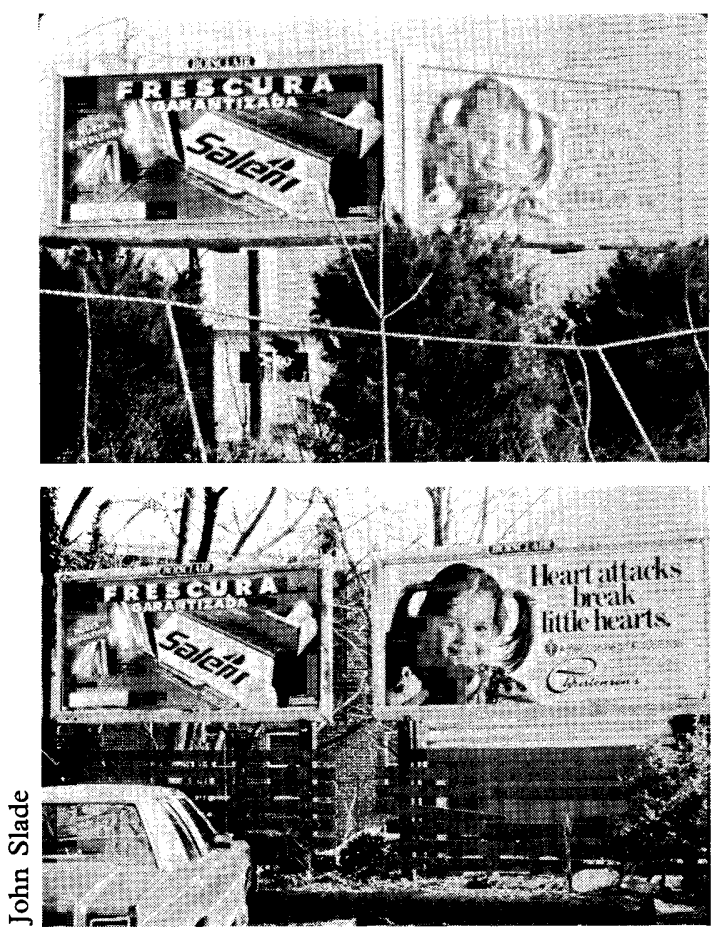

All the American Heart Association's public service billboards observed in Perth Amboy, New fersey, USA in fanuary 1992 were paired with promotions for the leading cause of cardiovascular disease.
15 Gutzwiller F, La Vecchia C, Levi F, Negri E, Wietlisbach V. Smoking, prevalence of disease and health service utilization among the Swiss population. Rev Epidemiol Sante Publ 1989; 37: 137-42.

16 Rosenberg L, Kaufman DW, Helmrich SP, Shapiro S. The risk of myocardial infarction after quitting smoking in men under 55 years of age. $N$ Engl 7 Med 1985; 313: 1511-14.

17 Rosenberg L, Kaufman DW, Helmrich SP, Miller DR Stolley PD, Shapiro S. Myocardial infarction and cigarette smoking in women younger than 50 years of age. cigarette smoking in women

18 Rosenberg L, Palmer JR, Shapiro S. Decline in the risk of myocardial infarction among women who stop smoking. N Engl f Med 1990; 322: 213-7.

19 Willett WC, Green A, Stampfer MJ, et al.: Relative and absolute excess risks of coronary heart disease among women who smoke cigarettes. $N$ Engl f Med 1987; 317: 1303-9.

20 Gramenzi A, Gentile A, Fasoli $M$, et al. Smoking and myocardial infarction in women: A case-control study from Northern Italy. F Epidemiol Commun Health 1989; 43: 214-7.

21 Bruzzi P, Green SB, Byar DP, Brinton LA, Schairer C. Estimating the population attributable risk for multiple risk factors using case-control data. Am F Epidemiol 1985; 122: 904-14.
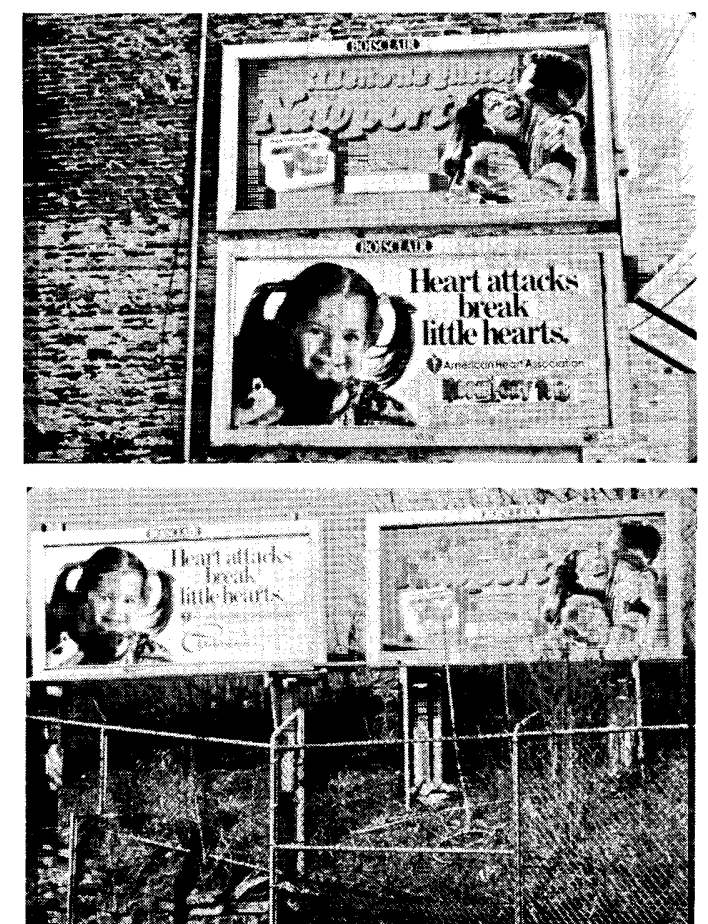